\title{
ESTUDO DESCRITIVO SOBRE A DEMANDA DE UM PRONTO-ATENDIMENTO PSICOLÓGICO EM UM SERVIÇO-ESCOLA
}

\author{
Sarah Rabelo de Souza, Heidi M. Bertolucci Coelho, Walter José Martins Migliorini \\ Universidade Estadual Paulista - UNESP, Faculdade de Ciências e Letras, Assis, SP. Agência de fomento: PROEX - Pró- \\ Reitoria de Extensão Universitária. E-mail: sarahrs@femanet.com.br
}

\section{RESUMO}

O Projeto de Extensão de Pronto Atendimento do Centro de Pesquisa e Psicologia Aplicada (CPPA) da UNESP - Assis tem como objetivo oferecer à comunidade discente um plantão para o acolhimento psicológico e possíveis encaminhamentos para psicoterapia. O objetivo deste trabalho foi o de apresentar dados sociodemográficos (sexo, idade, curso, seriação, profissão, encaminhamento) relativos ao perfil da clientela deste projeto no biênio 2012-2013. A metodologia utilizada foi análise documental, e os dados foram coletados diretamente das fichas de triagem. Foi realizada análise estatística descritiva utilizando os softwares Excel e Bioestat. Os resultados apontaram para um aumento na demanda deste serviço, contando com maior procura por jovens ingressantes no curso superior e que não exerciam atividade remunerada. Esta pesquisa exploratória possibilitou elaborar um perfil inicial da clientela do Pronto Atendimento e deu continuidade aos estudos já realizados anteriormente.

Palavras Chave: clínica-escola de psicologia, psicologia preventiva, psicoterapia.

\section{DESCRIPTION STUDY ABOUT THE DEMAND OF A READY-SERVICE PSYCHOLOGICAL IN A SERVICE- SCHOOL.}

\begin{abstract}
The Extension Project of a ready-service Psychological of Centro de Pesquisa e Psicologia Aplicada (CPPA) , UNESP - Assis aims to offer the students, psychological care and possible referrals to the psychotherapy. The objective of this study was to present and compare demographic data (gender, age , travel, serialization , profession, routing ) for the client profile in the biennium 20122013. The methodology used was documentary analysis, and data were collected directly from screening records. Descriptive statistical analysis was performed using Excel and Bioestat software. The results pointed to an increase in demand for this service, with greater demand for young freshman in college with not paid activity. This exploratory research enabled prepare an initial profile of the clientele of the psychological care and continue the studies conducted earlier. Keywords: clinical school of psychology, preventive psychology, psychotherapy.
\end{abstract}




\section{INTRODUÇÃO}

As clínicas-escola de Psicologia, ou serviços-escola, como descritas por Cavalheiro et al. (2012), podem oferecer à população, principalmente a economicamente desfavorecida, o acesso aos serviços psicológicos gratuitos, exercendo, portanto, um papel social importante na comunidade em que a Universidade está inserida, seja interna ou externa. Além disso, nos diferentes núcleos de estágios, os alunos do curso de Psicologia têm a possibilidade de aplicar seus conhecimentos teóricos à prática clínica, seja por meio dos atendimentos psicológicos individuais ou em grupos, como pela participação nas sessões de supervisão e nos grupos de estudos teóricos. Estas atividades acabam contribuindo para uma melhor formação de psicólogos. O Centro de Pesquisa e Psicologia Aplicada (CPPA), vinculado à Faculdade de Ciências e Letras da UNESP - Assis oferece serviços de clínica-escola sendo, portanto, o local onde os alunos do curso de Psicologia desta instituição podem realizar atividades práticas concernentes à área da psicologia clínica.

A maior procura pelos serviços da clínica-escola do Centro de Pesquisa e Psicologia Aplicada (CPPA) é oriunda da população externa. Porém, pensando no aumento da demanda dos estudantes da faculdade por acolhimento psicológico, foi criado em 1998 o Programa de ProntoAtendimento Psicológico ao Aluno (PPAPA) "com o intuito de oferecer à comunidade discente da unidade, uma possibilidade de acolhimento psicológico mediante a abertura de um lugar de escuta clínica" (PERES, SANTOS E COELHO, 2003, p. 48). Posteriormente, este projeto se tornou um Projeto de Extensão intitulado Programa de Pronto-Atendimento à Comunidade Local (conhecido como P.A.) e teve o auxílio financeiro da Pró-Reitoria de Extensão (PROEX). Neste Programa, os atendimentos são realizados gratuitamente por alunos dos 4으 e 5 을 anos do curso de Psicologia e têm duração de 50 minutos. As supervisões - clínicas e teóricas - são realizadas semanalmente com enfoque psicanalítico e são supervisionados por uma psicóloga do CPPA ou por um docente do curso de Psicologia. Nos anos de 2012 a 2014, aproximadamente 15 alunos estagiários estiveram envolvidos neste projeto, sendo oferecidas em média 30 horas semanais de plantão psicológico, e aproximadamente 65 sessões de psicoterapia individual para a população a que se destina.

Os estagiários deste projeto realizam aproximadamente duas horas semanais para os plantões e triagem e outros horários para realizarem entrevistas devolutivas e psicoterapia individual. Após a entrevista inicial de triagem, o estagiário relata o caso na supervisão semanal e um encaminhamento é proposto, podendo ser a psicoterapia individual na própria clínica-escola ou com profissionais conveniados. 
Uma característica peculiar deste projeto é que os atendimentos psicológicos aos estudantes do campus são realizados por outros estudantes, alunos do curso de Psicologia, sendo de extrema importância as recomendações realizadas nas supervisões sobre o sigilo profissional. Nesse sentido, quando é um aluno do próprio curso de Psicologia que procura por ajuda,ele é encaminhado, preferencialmente, para os psicólogos contratados pelo próprio Centro de Pesquisa e Psicologia Aplicada (CPPA) ou para profissionais da comunidade que mantêm convênio com a UNESP.

Além de propiciar o aprendizado da técnica na prática da clínica psicanalítica aos futuros psicólogos, o Pronto-Atendimento (P.A.) possibilita momentos de reflexão sobre saúde mental e a importância da escuta da dor psíquica. A escuta é fundamental para os estudantes que procuram o atendimento neste plantão psicológico em momentos de intensificação de suas angústias. Para Rocha (2004, p. 122), o plantão-psicológico é um momento de escuta,

(...) de estar presente num determinado momento e hora, mas, sobretudo, é estar aberto e disponível para receber e escutar o outro que procura por ajuda psicológica. (...) a escuta é a principal oferta do facilitador. Ouvir a pessoa que traz um problema, como ela está, como lida com o que a incomoda, que alternativas está podendo vislumbrar ou não.

A intervenção do terapeuta pode ser realizada a partir desta escuta. Rocha (2012) descreve como modelo tradicional de triagem aquele baseado em coletar os dados pessoais do cliente, identificar a queixa e realizar um breve diagnóstico. Para essa mesma autora, o processo de triagem tem sofrido reformulações e atualmente são utilizadas também as entrevistas de natureza, a um só tempo, diagnóstica e interventiva, a chamada de "triagem interventiva". Ancona-Lopes (1995) considera que toda atuação psicológica "é uma ação de intervenção cujo significado será dado pelo campo relacional que se estabelece entre as partes e que é exclusivo e peculiar àquele momento e àquela situação" (ANCONA-LOPES, 1995, p. 28).

Um perfil sociodemográfico da clientela

O presente trabalho procura apresentar alguns dados relativos ao perfil de usuários deste Programa de Ponto-Atendimento. A escolha dos anos considerados para este trabalho, 2012-2013 deve-se à relevância, pois nestes dois anos foi possível coletar dados de todo o período, o que não seria possível sobre os dados de 2014, devido à paralisação das atividades do CPPA durante o período de greve dos diversos segmentos na Universidade. Desta forma, estes foram os últimos anos em que este projeto foi desenvolvido sem interrupção até a presente data e, por essa razão, escolhidos para serem analisados. 
O objetivo deste trabalho, portanto, é o de apresentar dados sociodemográficos relativos ao perfil da clientela do Programa de Pronto-Atendimento Psicológico do Centro de Pesquisa e Psicologia Aplicada - UNESP, Assis, no biênio 2012-2013, considerando: sexo, idade, curso universitário, seriação e atividade profissional.

\section{METODOLOGIA}

Esta pesquisa é de natureza exploratória e a metodologia utilizada foi a análise documental (CAMPOS, 2001). Os dados sociodemográficos - variáveis quantitativas e qualitativas - foram coletados diretamente das fichas de triagem dos pacientes atendidos no biênio 2012-2013, organizados e analisados com ferramentas da Estatística Descritiva ${ }^{1}$. Para tanto, foram utilizados softwares Microsoft Excel e Bioestat (AYRES et al., 2007). Neste trabalho não foram realizados testes com objetivos inferenciais ${ }^{2}$.

\section{RESULTADOS}

Os resultados apontaram um aumento de aproximadamente $15 \%$ da procura pelo plantão neste período. Em 2012 foram 112 atendimentos e em 2013 foram 129. No início do programa, ocorrido em 1998, houve a procura de 28 estudantes $^{3}$. A Figura 1 mostra aumento na procura.

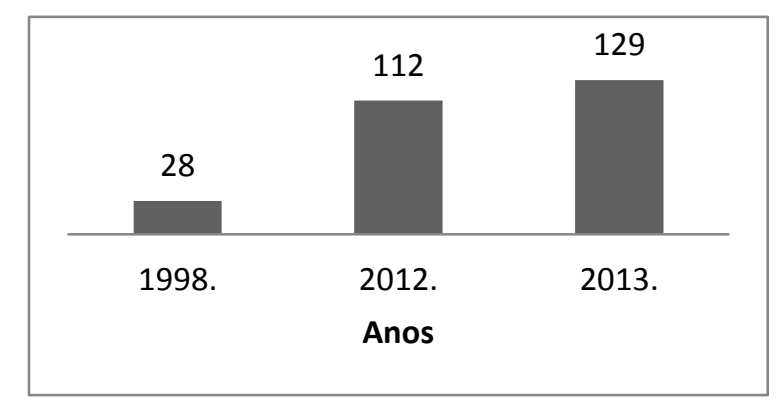

Figura 1. Número de usuários, em função do ano, do P.A.- CPPA, UNESP - Assis.

A média de idade em 2012 foi de 22,4 anos ( $s=4,1$ anos) e em 2013 de 21,6 anos (s=4,7 anos). A idade mínima foi de 17 anos nos dois anos (2012 e 2013) e a máxima foi de 41 anos em 2012 e 61 anos em 2013. Os resultados apontaram que houve uma maior porcentagem de estudantes com idade entre 17 e 21 anos (50,0\% em 2012 e 62,7\% em 2013) e a maior procura do

\footnotetext{
${ }^{1}$ Estatística Descritiva: "conjunto de técnicas destinadas a descrever e resumir os dados, a fim de que possamos tirar conclusões a respeito de características de interesse" (MAGALHÂES, LIMA, 2004, p. 2).

'Inferência Estatística: "é o estudo de técnicas que possibilitam a extrapolação, a um grande conjunto de dados, das informações e conclusões obtidas a partir de subconjuntos de valores, usualmente de dimensão muito menor" (MAGALHÂES, LIMA, 2004, p. 2).

${ }^{3}$ Dados de pesquisa realizada e publicada por Peres, Santos e Coelho (2004). 
serviço foi pelo sexo feminino (69,6\% em 2012 e 63,6\% em 2013). Os dados dessas variáveis idade e sexo - são apresentadas na Tabela 1.

Tabela 1. Sexo e idade de usuários do P.A.- CPPA, UNESP - Assis, biênio 2012-2013.

\begin{tabular}{l|l|l|l|l}
\hline Características & \multicolumn{2}{|l|}{2012} & \multicolumn{2}{l}{2013} \\
\hline Sexo & $\mathrm{N}$ & $\%$ & $\mathrm{n}$ & $\%$ \\
Feminino & 78 & 69.6 & 82 & 63.6 \\
Masculino & 34 & 30.4 & 47 & 36.4 \\
Total & 112 & & 129 & \\
Idade (anos) & & & & \\
de 17 a 21 anos & 56 & 50.0 & 81 & 62.7 \\
de 22 a 26 anos & 43 & 38.4 & 39 & 30.2 \\
de 27 a 31 anos & 8 & 7.1 & 5 & 3.9 \\
de 32 a 36 anos & 3 & 2.7 & 1 & 0.8 \\
\hline 37 ou mais anos & 2 & 1.8 & 2 & 1.6 \\
\hline Nada Consta & - & - & 1 & 0.8 \\
\hline
\end{tabular}

Considerando as idades inferiores ao terceiro quartil, ou seja, em $75 \%$ do total de idades observadas, houve uma maior concentração de alunos com idades menores em 2013 se comparada a 2012, como mostra a Figura 2.

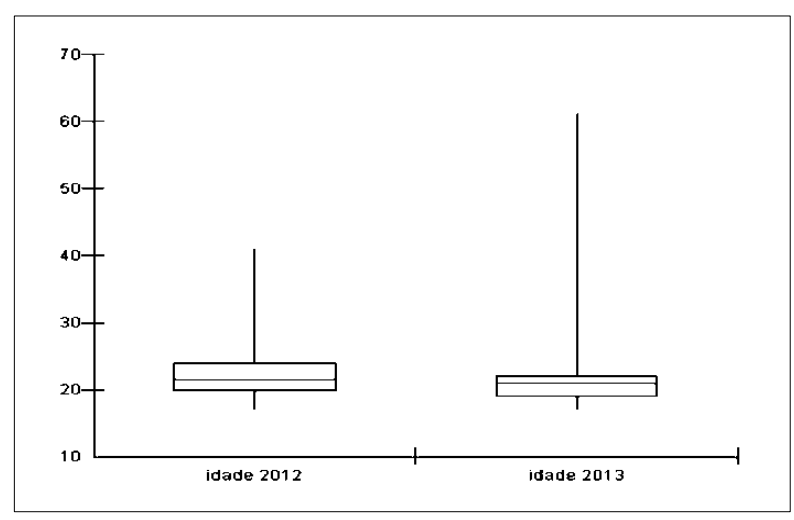

Figura 2. Box-plot das idades da clientela do P.A.- CPPA, UNESP - Assis, biênio 2012-2013.

A Figura 3 ilustra que grande parte dos estudantes (97\% em 2012 e 95\% em 2013) não exercia atividade profissional remunerada. 


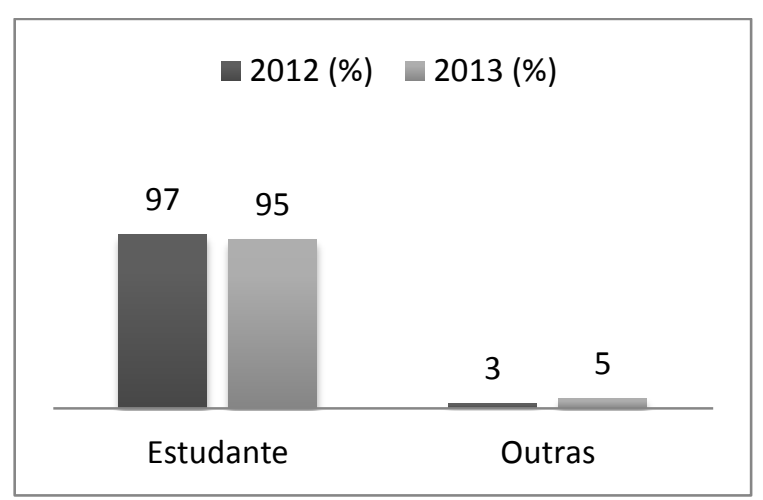

Figura 3: Profissão dos usuários do P.A. - CPPA - UNESP - Assis, biênio 2012-2013.

Foi verificada que, aproximadamente, 29\% dos usuários em 2012 e 25\% em 2013 cursavam o primeiro ano da faculdade (Figura 4).

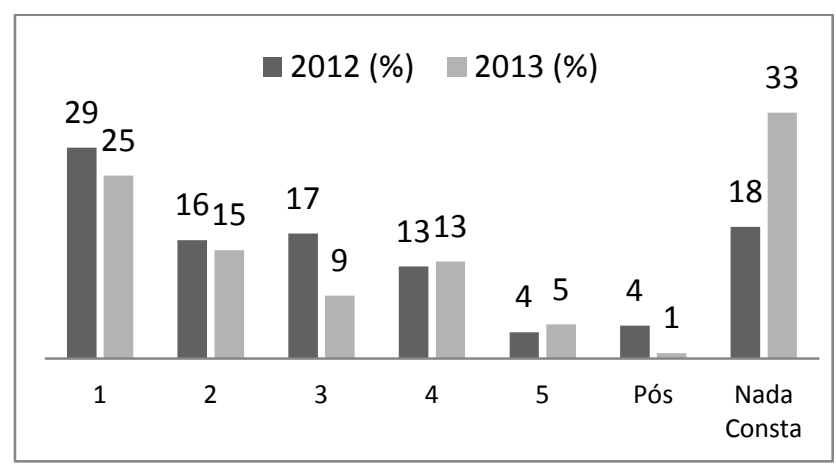

Figura 4: Seriação dos cursos dos da clientela do P.A. - CPPA - UNESP - Assis, biênio 2012-2013

\section{DISCUSSÃO}

O número de atendimentos é modesto se considerado o número de alunos matriculados no campus que, segundo o Anuário Estatístico da UNESP, foi de 1751 em 2012 e 2056 em 2013. Porém, deve-se enfatizar que o número de estudantes que procuram o atendimento no P.A. tem aumentado, sendo que em 1998, ano de início deste projeto, foram 28 usuários.

Verificou-se uma leve diminuição das idades dos estudantes no biênio estudado e em relação aos dados anteriores. Peres, Santos e Coelho (2004) observaram que apenas 2 alunos $(3,4 \%)$ em 2000 e 3 alunos (3,8\%) em 2001 tinha idades inferiores a 19 anos. Dos dados coletados nesta pesquisa, verificou-se que em 2012 foram 8,9\% (10 alunos) e em 2013, 10,1\% (13 alunos) com idades inferiores a 19 anos. Portanto, os dados apresentados parecem mostrar que a idade dos alunos que tem procurado este serviço de atendimento psicológico tem diminuído durante esses anos.

Os dados apontam que no biênio estudado houve uma maior procura por estudantes do sexo feminino. Esse resultado corrobora com os de Porto, Valente e Rosa (2014), que pesquisaram o 
perfil da clientela da comunidade externa da mesma Universidade - UNESP, Assis. Nesta pesquisa foi verificada que, entre os adultos a partir de 18 anos, a maior demanda nos anos de 2008 a 2012 foi de mulheres, sendo que em 2012 foram aproximadamente $76 \%$ do total da clientela. Apesar dos dados mostrarem uma procura maior pelo sexo feminino pelo Pronto-Atendimento, deve-se notar que houve um aumento de $6 \%$ na procura por usuários do sexo masculino. Considerando os dados de Peres, Santos e Coelho (2004), nota-se que houve um aumento significativo do público masculino pelo Pronto-Atendimento neste intervalo de tempo, já que em 2000 foram 17\% e em $2001,16 \%$.

Os resultados referentes às idades concordam com os da seriação no curso que os alunos estão matriculados, ou seja, a maioria dos alunos que procura o Pronto-atendimento é de alunos que acabaram de ingressar na universidade. Além disso, a maioria não possui atividade remunerada. Desta forma, pode-se considerar que, em geral, estes jovens se encontram num período final da adolescência, em transição a idade adulta. Calligaris (2013, p. 15) caracteriza este período de transição como um estado de moratória ${ }^{4}$, quando o jovem ainda não é reconhecido como adulto "apesar de seu corpo e seu espírito estarem prontos para a competição". Outeiral (2008) considera a adolescência em três etapas, sendo que na sua etapa final, de 17 a 20 anos, destacam-se elementos importantes, como o estabelecimento de novos vínculos com os pais, a escolha profissional e a aceitação do próprio corpo e dos processos psíquicos do mundo adulto.

Goldstein (2013) entrevistou profissionais renomados no campo da psicoterapia clínica psicanalítica e estes apontaram questões que os pacientes apresentam atualmente na clinica. Entre elas, podem-se destacar algumas que também são apresentadas pelos jovens usuários do Pronto-atendimento, como a solidão, os processos de desenraizamento, a crise dos papéis de autoridade, a depressão, a não aceitação do sofrimento - sistematicamente patologizado e medicalizado, na atualidade - a imersão no mundo virtual e seus efeitos, o aumento de comportamentos compulsivos e irrefletidos.

\section{CONCLUSÕES}

A procura por jovens universitários pelo Pronto-Atendimento psicológico aumentou no período de 2012-2013. Além disso, os resultados parecem mostrar que esses estudantes: (1) são majoritariamente, do sexo feminino, apesar de ter sido observado um aumento de estudantes do sexo masculino; (2) estão ingressando na universidade; (3) possuem idade, em sua maioria, abaixo de 21 anos com tendência decrescente e (4) grande parte deles não possui atividade remunerada.

\footnotetext{
${ }^{4}$ Moratória: Dilatação do prazo, prorrogação "que o credor concede ao devedor para o pagamento da divida. Adiamento dos vencimentos das dívidas, suspensão dos pagamentos e da ação da justiça (...)" (MICHAELIS, 2000, p.416).
} 
Pode-se concluir que esta pesquisa exploratória pode contribuir para conhecer o perfil inicial da clientela do Programa de Pronto Atendimento. Isso poderá auxiliar em tomadas de decisões para possíveis ações para melhorar o acolhimento do estudante ingressante nesta universidade. Além disso, este programa de extensão, voltado especificamente para o atendimento do jovem universitário, tem contribuído para a formação dos futuros psicólogos, já que nas supervisões, clínica e teórica, temas associados aos jovens e à subjetividade humana na contemporaneidade podem ser debatidos e pensados por todos.

Além disso, estes resultados complementam os estudos realizados por Peres, Santos, e Coelho, (2004) e possibilitam que novas análises comparativas sejam realizadas futuramente.

\section{REFERÊNCIAS}

ANCONA-LOPEZ, M. Psicodiagnóstico: processo de intervenção? In: ANCONA-LOPEZ, M. (Org.). Psicodiagnóstico: Processo de intervenção. São Paulo: Cortez, 1995, p. 26-36.

ANUÁRIO ESTATÍSTICO: Universidade Estadual Paulista "Júlio de Mesquita Filho". São Paulo: UNESP, APE, 2001. Disponível em: https://ape.unesp.br/anuario/pdf/Anuario_2015.pdf Acesso em: 18/08/2015.

AYRES, M.; AYRES-Jr, M.; AYRES, D.L.; SANTOS, A.A.S. Bioestat: aplicações estatísticas nas áreas das Ciências Biomédicas. Versão 5.0. Belém, Pará: Sociedade Civil Mamirauá, MCT-CNPq, 2007.

CALLIGARIS, C. A adolescência. São Paulo: Publifolha, 2009.

CAMPOS, L. F. L. Métodos e técnicas de pesquisa em psicologia. Campinas: Editora Alinea, 2001.

CAVALHEIRO, N. C. et al. Triagem interventiva: a caracterização de uma demanda. Rev. SBPH, vol. 15, n. 2, Rio de Janeiro, Jul/Dez, 2012.

GOLDSTEIN, T. S. Psicologia e mundo contemporâneo: o que quer e o que pode essa clínica?. 2013, 213 f. Tese (Doutorado). Instituto de Psicologia, Universidade de São Paulo, São Paulo, S.P.

MAGALHÃES, M. N.; LIMA, A. C. P. Noções de Probabilidade e Estatística. São Paulo: EDUSP, 2004.

MICHAELIS: minidicionário escolar da língua portuguesa. São Paulo: Melhoramentos, 2000.

OUTEIRAL, J. Adolescer. Rio de Janeiro: Ed. Revinter, 2008.

PERES, R. S.; SANTOS, M. A.; COELHO, H. M. B. Atendimento psicológico a estudantes universitários: considerações acerca de uma experiência em clínica-escola. Estud. psicol. (Campinas), Campinas, v. 20, n. 3, Dec. 2003 Disponível em $<$ http://www.scielo.br/scielo.php?script=sci_arttext\&pid=S0103$166 \times 2003000300004 \&$ Ing=en\&nrm=iso $>$.Acessado em 29 Abr. 2015. http://dx.doi.org/10.1590/S0103-166X2003000300004 
PERES, R. S.; SANTOS, M. A.; COELHO, H. M. B. Perfil da clientela de um programa de prontoatendimento psicológico a estudantes universitários. Psicol. estud., Maringá , v. 9, n. 1, Abril 2004.Disponível em <http://www.scielo.br/scielo.php?script=sci_arttext\&pid=S141373722004000100007\&lng=en\&nrm=iso> Acesso em 29 Abr. 2015.

PORTO, M. A.; VALENTE, M. L. L.; ROSA, H. R. A construção do perfil da clientela numa clínicaescola. Boletim de Psicologia: Sociedade de Psicologia de São Paulo, São Paulo, v. LXIV, n. 141, jul/dez 2014. P. 159-172.

ROCHA, M. C. Plantão psicológico e triagem: aproximações e distanciamentos. Rev. NUFEN, São Paulo, v.3, n.1, 2011.Disponível_em

$<$ http://pepsic.bvsalud.org/scielo.php?script=sci_arttext\&pid=S2175-

$25912011000100007 \&$ lng=pt\&nrm=iso>. Acesso em 24 jul. 2015. 